\title{
Radial Basis Function Differential Quadrature for Hydrodynamic Pressure on Dams with Arbitrary Reservoir and Face Shapes Affected by Earthquake
}

\author{
A. M. Behroozi and M. Vaghefi ${ }^{\dagger}$ \\ Department of Civil Engineering, Persian Gulf University, Shahid Mahini St., Bushehr 75169, Iran \\ †Corresponding Author Email: Vaghefi@pgu.ac.ir
}

(Received December 29, 2019; accepted April 17, 2020)

\begin{abstract}
A mesh-free numerical model based on the Radial Basis Function Differential Quadrature is introduced to simulate the hydrodynamic response of the dam-reservoir-foundation system affected by earthquake acceleration. The governing equation of the hydrodynamic pressure of dam-reservoir system with non-vertical face shape was discretized using the present model. Different formulation were implemented to model far-end of unbounded domain as boundary condition. For this purpose, the effects of fluid compressibility and energy depreciation in the reservoir boundaries were considered simultaneously. The present model was used to determining the distribution of hydrodynamic pressure on dam face caused by earthquake acceleration in several practical examples and the obtained results were compared with available well-known analytical solutions. The comparison indicate that the accuracy and efficiency of the present model are quite satisfactory.
\end{abstract}

Keywords: Dam-reservoir interaction; Radial basis function differential quadrature; Mesh-free method; Hydrodynamic pressure; Computation cost.

\section{NOMENCLATURE}

$\begin{array}{ll}a & \text { acceleration } \\ c & \text { shape factor } \\ C_{w} & \text { velocity of the sound wave in water } \\ H & \text { reservoir depth } \\ L_{2} & \text { the norm of relative error } \\ p & \text { hydrodynamic pressure } \\ x, y & \text { cartesian coordinates } \\ W_{x}^{n} & \text { coefficients of n-th order derivatives with } \\ & \text { respect to } \mathrm{x}\end{array}$

\section{INTRODUCTION}

Determining the distribution of hydrodynamic pressures on the upstream surface of the earthquakeaffected dam as one of the most challenging issues in the water field of fluid-structure interaction is an important factor in designing dams in seismic areas. Thus several approaches and methods have been documented in the literature for capturing the hydrodynamic response of hydraulic structures when affected by earthquake acceleration. Westergaard (1933) first analytically solved the hydrodynamic pressure on vertical face dams when subjected to

\author{
$W_{y}^{n} \quad$ coefficients of $\mathrm{n}$-th order derivatives with \\ respect to $\mathrm{y}$ \\ $\alpha \quad$ damping coefficient of reservoir bottom \\ $\beta \quad$ reservoir bottom slope \\ $\theta$ dam face inclination \\ $\rho \quad$ water density \\ $\Psi \quad$ multiquadric Radial basis function
}

horizontal ground acceleration, by assuming the water to be incompressible. Since then many researchers have tried to cover Westergaard's method limitation such as considering fluid compressibility and complexity of reservoir, dam face shape (Chopra 1967; Chwang and Housner 1978; Liu 1986; Bouaanani et al. 2003). However, they were all limited to some simplifying assumptions and for more complicated problems like complex geometries with considering the effect of energy absorption of the reservoir bottom, needs numerical approaches to investigate the reservoirdam interaction completely. Several researchers 
have developed more advanced numerical approaches for simulating seismic behavior of damreservoir system during earthquake. Finite difference method was used in (Nath 1971; Hung and Wang 1987; Wang and Hung 1990; Chen and Hung 1993; Abdollahi and Attarnejad, 2012). The Finite Element approach (FEM) has often been the most widely used method in this field. The Eulerian and Lagrangian fluid finite element method was adopted in (Saini et al. 1978; Wilson and Khalvati 1983). Hall and Chopra (1982) presented a finite element based numerical solution in the frequency domain for computing the hydrodynamic pressure on the concrete and embankment dam accelerated by ground motion. Later Fenves and Chopra (1984) and Lotfi et al. (1987) extended the previous work to take account of the effects of energy absorbing of reservoir bottom due to alluvium and sediments. The coupling the finite element method in solid and fluid domain with considering effects of energy absorption of reservoir bottom on the solution was proposed to analysis the hydrodynamic response of elastic dam in time-domain by Küçükarslan et al. (2005). Bouaanani and Lu (2009) ignored viscosity effects and discretized potential-based governing equation of fluid using finite element method to study dam-reservoir interaction under earthquake. The finite element method in the time domain was used by Maity and Bhattacharyya (1999); Gogoi and Maity (2006). Boundary Element Method (BEM) in the frequency domain was used for investigation the dynamic response of concrete gravity dams and interactions of dam-reservoir-foundation by (Humar and Jablonski 1988; Medina and Domínguez 1989; Wang, Lin and Hu, 2015; Wang, Hu and Guo, 2019). A numerical approach based on coupling FEM and BEM also adopted for simulating problems related to fluid-structure interaction by (Estorff and Antes 1991; Belytschko and Lu 1994; Czygan and von Estorff, 2002). The displacement fluid finite elements was used to simulate gravity dam-reservoir interaction and comparison of stochastic and deterministic dynamic responses of system by Bayraktar et al. (2005). Pelecanos et al. (2013) studied rigid and flexible dams under various acceleration loads by implementing different formulation for boundary conditions. Demirel (2015) adopted the Ghost-cell immersed boundary method to determine the hydrodynamic pressure distribution on dams with curved and non-vertical face under earthquake acceleration.

Recently coupled FEM and BEM approach was used for modeling water structure interaction in frequency domain (Zhao et al., 2019; Zhao et al., 2020)

The main objective of this work is to use the Radial Basis Function Differential Quadrature as a meshfree numerical model to solve the potential-based governing equation of dam-reservoir systems under steady-state and time-dependent acceleration loads. This paper is outlined as follows: The section 2 is assigned to a brief description of the governing equation of hydrodynamic response of the damreservoir system under earthquake acceleration and also describing initial and boundary conditions. The numerical formulation of RBF-DQM is introduced in section 3. The discretization process of governing equation and boundary conditions and implementation the present algorithm are presented in sections 4, 5 respectively. Various case studies are solved by RBF-DQM for evaluation of model efficiency, in section 6 and finally, section 7 is dedicated to the conclusion.

\section{HydRODYNAMIC GOVERNING EQUATION OF RESERVOIR}

The hydrodynamic pressure $p$ in the constant depth reservoir behind a dam affected by the earthquake acceleration as illustrated in Fig. 1 is expressed by the acoustic wave equation as follow:

$\nabla^{2} p=\frac{1}{c_{w}{ }^{2}} \frac{\partial^{2} p}{\partial t}$

Where $c_{w}$ is the velocity of the sound wave in water (Fenves and Chopra 1984). In the Eq. (1) the fluid is considered compressible and having negligible viscosity.

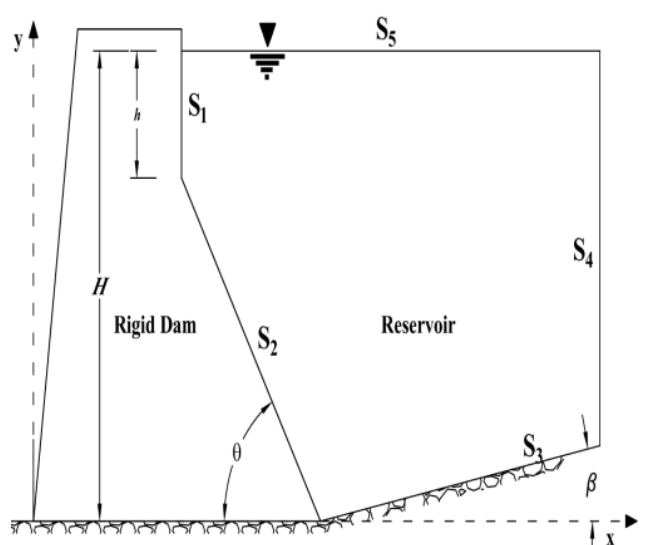

Fig. 1. Schematic of Dam-reservoir System.

In the dam-reservoir interface (S1, S2) satisfying the stress continuity condition as follow (Liu 1986):

$\frac{\partial p}{\partial \vec{n}}=-\rho a_{n}$

In which $\vec{n}$ represents the unit vector perpendicular to the interface boundaries, $a_{n}$ is the normal component of acceleration vector on the interfaces and $\rho$ is fluid density.

In the previous study the, reservoir-foundation interaction (S3) is mainly modeled by using two formulation as boundary conditions. With neglecting the effects of the energy dissipation at the reservoir bottom, fully reflective boundary condition can be used to model the reservoir-bottom interaction as follow (Liu 1986):

$\frac{\partial p}{\partial \vec{n}}=-\rho a_{n}$

By taking into account the effect of energy dissipation of reservoir bottom, following boundary condition can be forced: 
$\frac{\partial p}{\partial \vec{n}}=-\rho a_{n}-\tilde{q} \frac{\partial p}{\partial t}$

$\tilde{q}$ is damping parameter to characterize the effects of the alluvial reservoir bottom. Flowing equation was presented by Fenves and Chopra (1984) to express it:

$\tilde{q}=\frac{1-\alpha}{c_{w}(1+\alpha)}$

In which $\alpha$ is dimensionless parameter shows which represents the ratio of the amplitudes of the reflected and induced compressive wave related to reservoir bottom. $\alpha$ is variable between 0 and 1 for nonreflective and 1 fully reflective reservoir bottom respectively.

Various formulations have been proposed for modeling the far end of reservoir (S4) where the unbounded domain is truncated. In the simplest form following expression can be applied:

$\lim _{x \rightarrow \infty} p=0$

In order to optimize computation cost, the infinite reservoir must truncate at a reasonable distance. For this purpose the Sommerfeld-type radiation boundary condition as introduced by Hung and Wang (1987) can be implemented, at truncated boundary as follows:

$\frac{\partial p}{\partial x}=-\frac{1}{c_{w}} \frac{\partial p}{\partial t}$

Sharan (1987), in order to shorten the truncating distance of the unbounded fluid domain and consequently reduce the computational cost, by ignoring the effects of viscosity and compressibility of fluid and using a damper technique, developed another formulation for modeling far end of reservoir as follow:

$\frac{\partial p}{\partial x}=-\frac{\pi}{2 H} p-\frac{1}{c_{w}} \frac{\partial p}{\partial t}$

At the free surface of fluid (S5) short amplitude wave on the water free surface can be ignored due to their neglect able height compared to the depth of the reservoir. Thus the boundary condition of the free surface can be implemented as follow:

$p=0$

In initial state the reservoir is in rest, therefore, the initial conditions are:

$\left.\mathrm{p}\right|_{\mathrm{t}=0}=0,\left.\frac{\partial \mathrm{p}}{\partial \mathrm{t}}\right|_{\mathrm{t}=0}=0$

\section{Methodology}

\subsection{Numerical Formulation of RBF-DQM Method}

The main theory of the differential quadrature method (DQM) method came from the quadrature integration, where the integral over a closed domain is approximated by a linear combination of function values at whole computation points of domain. The (DQM) is a numerical discretization approach that approximates the derivative of a function $f(x, y)$ with respect to $\mathrm{x}$, using a linear combination of function values in the domain in $\mathrm{x}$-direction (Bellman and Casti 1971) as follow:

$f_{x}^{n}\left(\boldsymbol{x}_{i}\right)=\sum_{j=1}^{N} W_{x}^{n}(i, j) \cdot f\left(\boldsymbol{x}_{j}\right)$

for $i=1,2, \ldots, N$

For the derivative corresponding to $\mathrm{y}$, is also written:

$f_{y}^{n}\left(\boldsymbol{x}_{i}\right)=\sum_{j=1}^{N} W_{y}^{n}(i, j) \cdot f\left(\boldsymbol{x}_{j}\right)$

for $i=1,2, \ldots, N$

Where $W_{x}^{n}, W_{y}^{n}$ are coefficients of $\mathrm{n}$-th order derivatives with respect to $\mathrm{x}$ and $\mathrm{y}$, and $N$ is number of mesh points. Varieties of methods have been developed based on the DQM method, including the polynomial-based differential quadrature (PDQ) and the Fourier-expansion-based differential quadrature (FDQ) (Shu and Richards 1992; Shu and Chew 1997).

In the PDQ, the coefficients of derivatives are determined using an algebraic expressions or a recurrence relationship that is independent of the selection of the nodal points. In a similar approach, the FDQ uses the Fourier series expansion to approximate the function.

Although the PDQ and FDQ methods are high accurate with low computational effort, they are mesh-based methods (Shu, 2000). Wu and Shu (2002) applied radial basis functions (RBF) to the DQM method to take advantage of the naturally meshless method that RBFs provide. In the RBFDQM method, the coefficients are determined using radial basis functions. In this method, the interplant to the function $\mathrm{f}$ at each mesh point $\boldsymbol{x}_{i}=\left(x_{i}, y_{i}\right)$ can be written as:

$f\left(x_{i}\right)=\sum_{k=1}^{N} \lambda_{k} \Psi_{k}\left(x_{i}\right)$

Where $\lambda_{k}$ is the coefficient for $\Psi_{k}\left(\boldsymbol{x}_{i}\right)$ and $f$ is a RBF. The RBFs can be used in various forms. The Multiquadric Radial basis function (MQ-RBF) is widely used because of better efficiency in interpolation of the 2-dimensional scattered data (Ding et al. 2005). The MQ-RBF is expressed as follow:

$\Psi_{k}(\boldsymbol{x})=\left\|\boldsymbol{x}-\boldsymbol{x}_{k}\right\|=$
$\sqrt{\left(x-x_{k}\right)^{2}+\left(y-y_{k}\right)+c^{2}} \quad c>0$

The unknown weighting coefficients can be determined by solving following linear system of equations:

$\frac{\partial^{n} \Psi_{k}\left(\boldsymbol{x}_{i}\right)}{\partial x^{n}}=\sum_{j=1}^{N} W_{x}^{n}(i, k) \Psi_{k}\left(\boldsymbol{x}_{i}\right)$

Noted that the values of RBF and also their $n$-th order derivatives are known at each computation points. Eq. (16) can be summarized in the matrix form as follow: 
A. M. Behroozi and M. Vaghefi / JAFM, Vol. 13, No. 6, pp. 1759-1768, 2020.

Table 1 Discretization the Boundary Conditions

\begin{tabular}{|c|c|c|}
\hline Boundary Type & $\begin{array}{c}\text { Boundary } \\
\text { No }\end{array}$ & Discretized Form \\
\hline The acceleration B/C Eq. (2) & $\mathrm{S} 1$ & {$\left[\mathrm{w}_{\mathrm{x}}^{(1)}\right]\{\mathrm{p}\}^{\mathrm{t}+1}=$ p.a. $\{1\}^{*}$} \\
\hline The acceleration B/C Eq. (2) & S2 & {$\left[\sin \theta \cdot \mathrm{w}_{\mathrm{x}}^{(1)}+\cos \theta \cdot \mathrm{w}_{\mathrm{y}}^{(1)}\right]\{\mathrm{p}\}^{\mathrm{t}+1}=\rho \cdot \mathrm{a} \cdot \sin \theta \cdot\{1\}$} \\
\hline The Rigid Bottom B/C Eq. (3) & S3 & $\left.\left[\sin \theta \cdot w_{\mathrm{x}}^{(1)}+\cos \theta \cdot \mathrm{w}_{\mathrm{y}}^{(1)}\right]\right]\{\mathrm{p}\}^{\mathrm{t}+1}=\rho \cdot \mathrm{a} \cdot \sin \theta \cdot\{1\}$ \\
\hline $\begin{array}{l}\text { The Flexible Bottom B/C Eq. } \\
\text { (4) }\end{array}$ & S3 & 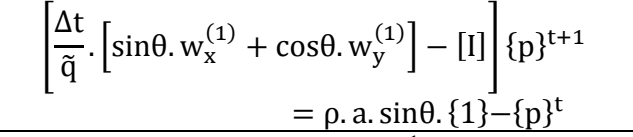 \\
\hline Sommerfeld-type B/C Eq. (7) & S4 & {$\left[\mathrm{w}_{\mathrm{x}}^{(1)}-\mathrm{I}\right]\{\mathrm{p}\}^{\mathrm{t}+1}=\frac{1}{\mathrm{c}_{\mathrm{w}} \cdot \Delta \mathrm{t}}\{\mathrm{p}\}^{\mathrm{t}}$} \\
\hline Sharan-type B/C Eq. (8) & S4 & {$\left[\mathrm{c}_{\mathrm{w}} \cdot \Delta \mathrm{t} \cdot\left[\mathrm{w}_{\mathrm{x}}^{(1)}\right]-\left(1+\frac{\mathrm{c}_{\mathrm{w}} \cdot \Delta \mathrm{t} \cdot \pi}{2 \mathrm{H}}\right)[\mathrm{I}]\right]\{\mathrm{p}\}^{\mathrm{t}+1}=\{\mathrm{p}\}^{\mathrm{t}}$} \\
\hline
\end{tabular}

$*\{1\}$ is $(\mathrm{M} \times 1)$ identity matrix and $\mathrm{M}$ is the number of mesh points on the Boundary

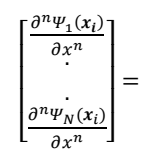

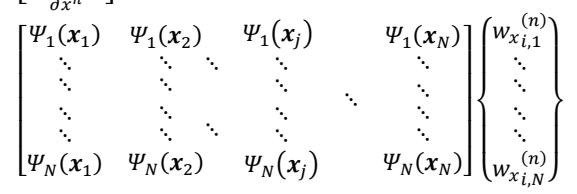

Similarly, the coefficients of higher order derivatives and the derivative coefficients in relation to other variables are also calculated in the same way. The center points $\boldsymbol{x}_{i}$ and $\boldsymbol{x}_{j}$ are called the Reference and the Supporting node respectively. $N$ is also defined as the number of supporting node in the approximation. If $N$ is considered as total mesh points, the model is called Global Radial Based Function Differential Quadrature, otherwise, if it is selected as limited numbers of mesh points in the neighborhood of the Reference node, the model is called Local Radial based Function Differential Quadrature. Fig. 2 is a schematic illustration for the arrangement of nodes in the computation domain. In present model, in contrast to mesh-base numerical method, instead of the connectivity between the mesh points, the Euclidean distance of points is important for discretization of PDE (Wu and Shu 2002). $c$ in Eq. (14) is a constant number called Shape Factor, which is depended on domain geometry and the distribution of the mesh points. Choosing a deviant value for $c$ decreases the accuracy of the numerical model (Wu and Shu 2002). In the current study, the value of $c$ is chosen based on the model presented by Hardy (1971) as follow:

$c=\frac{0.815}{N} \sum_{i=1}^{N} d_{i}$

Where $d_{i}$ is equal to the Euclidean distance of $i$-th mesh point to the nearest point in its neighborhood.

\section{Discretization OF The GOVERNING EQUATION}

The spatial derivatives of the partial differential Eq.
(1) in $i$-th mesh point is discretized using the RBFDQM as follow:

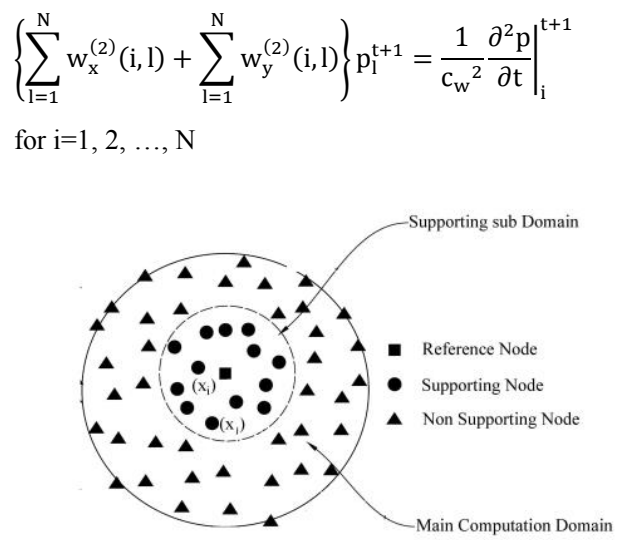

Fig. 2. Sketch for MQ-DQM Concept.

The first and second order temporal derivative pressure field which appear in governing equation and boundary conditions were discretized implicitly using the Forward and Central Finite Difference scheme respectively as follow:

$$
\begin{aligned}
& \left.\frac{\partial \mathrm{p}}{\partial \mathrm{t}}\right|_{\mathrm{i}} ^{\mathrm{t}+1}=\frac{\mathrm{p}_{\mathrm{i}}^{\mathrm{t}+1}-\mathrm{p}_{\mathrm{i}}^{\mathrm{t}}}{\Delta \mathrm{t}} \\
& \left.\frac{\partial^{2} \mathrm{p}}{\partial \mathrm{t}^{2}}\right|_{\mathrm{i}} ^{\mathrm{t}+1}=\frac{\mathrm{p}_{\mathrm{i}}^{\mathrm{t}+1}-2 \mathrm{p}_{\mathrm{i}}^{\mathrm{t}}+\mathrm{p}_{\mathrm{i}}^{\mathrm{t}-1}}{\Delta \mathrm{t}^{2}}
\end{aligned}
$$

Where $t-1, t$ and $t+1$ represent three consecutive time steps and $\Delta t$ is the time step. The Eqs. $(18,19)$ are summarized in matrix form as follow:

$$
\begin{aligned}
{\left[[\mathrm{I}]+\left(\Delta \mathrm{t} \cdot \mathrm{c}_{\mathrm{w}}\right)^{2}\left[\mathrm{w}_{\mathrm{x}}^{(2)}+\mathrm{w}_{\mathrm{y}}^{(2)}\right]\right]\{\mathrm{p}\}^{\mathrm{t}+1} } \\
=2\{\mathrm{p}\}^{\mathrm{t}}-2\{\mathrm{p}\}^{\mathrm{t}-1}
\end{aligned}
$$

In liner system of Eq. (20), $I(N \times N)$ is an identity matrix and $\left[w_{x}^{(2)}\right],\left[w_{y}^{(2)}\right]$ are coefficients matrix of second order derivative related to $x, y$, respectively. 
Table 2 Grid convergence Study for Case 1

\begin{tabular}{|c|c|c|c|c|c|}
\hline \multirow{3}{*}{$y / H$} & \multirow{2}{*}{ Tsai et al. (1990) } & \multicolumn{4}{|c|}{ Present Work (RBF-DQM) } \\
\cline { 2 - 6 } & & 212 & 322 & 388 & 437 \\
\cline { 2 - 6 } & & \multicolumn{5}{|c|}{$P^{*}$ on the upstream face } \\
\hline 0 & 0.7426 & 0.6418 & 0.6826 & 0.7419 & 0.7679 \\
\hline 0.2 & 0.7188 & 0.6247 & 0.6644 & 0.7222 & 0.7474 \\
\hline 0.4 & 0.6607 & 0.5704 & 0.6067 & 0.6595 & 0.6826 \\
\hline 0.6 & 0.5474 & 0.4727 & 0.5027 & 0.5464 & 0.5655 \\
\hline 0.8 & 0.3667 & 0.3134 & 0.3333 & 0.3623 & 0.3750 \\
\hline 1.0 & 0.0000 & 0.0000 & 0.0000 & 0.0000 & 0.0000 \\
\hline$L_{2}$ & - & $1.25 e-01$ & $7.52 e-02$ & $5.40 e-03$ & $3.01 e-02$ \\
\hline
\end{tabular}

Since Eq. (20) is implicitly discrete, it is unconditional stable, but conducting grid convergence study is necessary to reach the most accurate solution. The boundary conditions are also discretized, in a similar manner and summarized in Table 1.

\section{IMPLIMENTATIOM OF THE ALgORITEN}

The governing Eq. (1) and boundary conditions were discretized by the proposed algorithm. The MATLAB $^{\circledR}$ PDE MODELER toolbox was used to create and meshing the geometry (Howard, 2005). A MATLAB $^{\circledR}$ code and several MATLAB ${ }^{\circledR}$ functions were developed to implement the algorithm as previously formulated in Section 3, 4. The step by step implementation of the proposed algorithm is summarized as follows:

Step 1: Creating and meshing the computation domain and producing the matrix of mesh data using MATLAB $^{\circledR}$ PDE MODELER toolbox.

Step 2: forming and solving the system of linear Eq. (16) for determining derivative coefficients based on step 1 data.

Step 3: Forming and solving the system of linear Eq. (20) for time step $t+1$ base on the known pressure field in time step t. Boundary conditions as formulated in Table 1 also are forced in system of linear equations.

Step 4: Output pressure field for time step $\mathrm{t}+1$.

Step 5: Repeat this cycle from Step 3.

\section{RESULTS AND DISCUSSION}

To evaluate the efficiency of the RBF-DQM, two conceptual cases were simulated and the solution were compared with the well-known analytical solutions. In the first example, the hydrodynamic response of a non-vertical face dam with inclined reservoir, affected by steady-state horizontal acceleration was investigated. In the second case, the distribution of hydrodynamic pressure on a rigid gravity dam, due to harmonic and ramp acceleration was determined using the present model. The different boundary condition was implemented and the results were compared. In all cases, the fluid is specified by density $\rho=1000 \mathrm{~kg} / \mathrm{m} 3$ and velocity of the sound wave in water $c_{w}=1439 \mathrm{~m} / \mathrm{s}$. The dimensionless hydrodynamic pressure $P^{*}$ is also expressed as $P /\left(\rho a_{0} H\right)$ in which $a_{0}$ is the ground acceleration.

\subsection{Case1: Non-Vertical Face Rigid Dam}

Consider a dam with non-vertical face, as sketched in Fig. 1, consisting of an inclined section and a vertical free section. The dam was located on a steep reservoir that has constant depth $\mathrm{H}=100$ and extends to infinity $(x \rightarrow \infty)$.

The purpose of this hypothetical example was to investigate the capability of the RBF-DQM for determining the distribution of hydrodynamic pressure of an arbitrary shape dam face and sloping reservoir. The rigid dam-reservoir system is assumed to be affected with a constant acceleration of $a 0=1$ $\mathrm{m} / \mathrm{s}^{2}$. The effects energy depreciation of reservoir bottom and the low amplitude waves in free surface were neglected. Hence, the boundary condition of the reservoir bottom and free surface were assigned as Eq. (3) and Eq. (9) respectively. An analytical solution was introduced by Liu (1986) to such geometries. The reservoir was truncated in $\mathrm{L}=2 \mathrm{H}$ as the end far boundary.

To achieve the optimal mesh layout, the grid convergence study was performed on this case at $\beta=$ 0 and $\theta=90^{\circ}$. For this purpose, the results for different mesh numbers were compared with Liu (1986), which are summarized in Table 2 in which $\mathrm{L}_{2}$ (the norm of relative error) is used for computing error indicator as follow:

$L_{\mathbf{2}}=\sqrt{\frac{1}{s} \sum_{l}^{s}\left(\frac{u_{\text {exact }}-u_{R B F-D Q M}}{u_{\text {exact }}}\right)^{2}}$

Where $U_{R B F-D Q M}$, uexact and $s$ are the results from the proposed model, the analytical solution, and the number of sample points respectively (Shu et al. 2004).

Thus, based on this process, mesh layout with 388 computational nodes was considered as the optimal discretized domain in this case. Fig. 3 Portrays typical unstructured meshing layout for the case. At first, the free section height $h$ was considered zero $(h / H=0)$ and the hydrodynamic pressure for different value of dam face and reservoir bottom inclination 


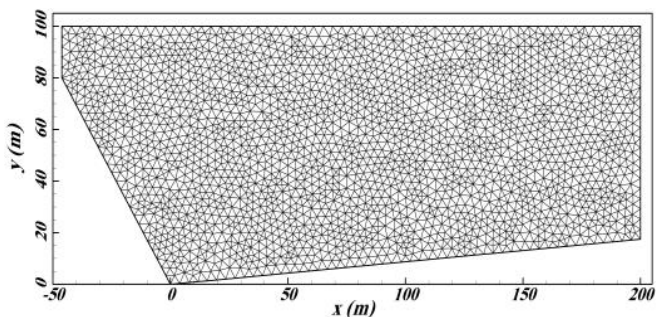

Fig. 3. Unstructured mesh points distribution of the computation domain for Case No.1.

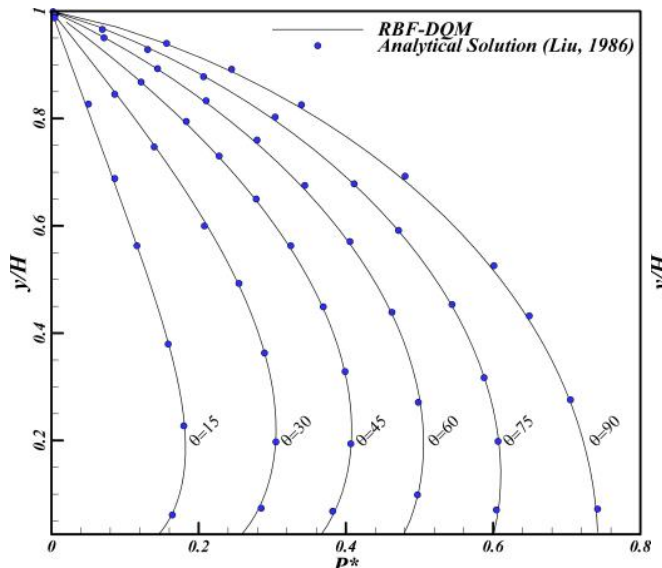

(a) $\beta=0^{\circ}$

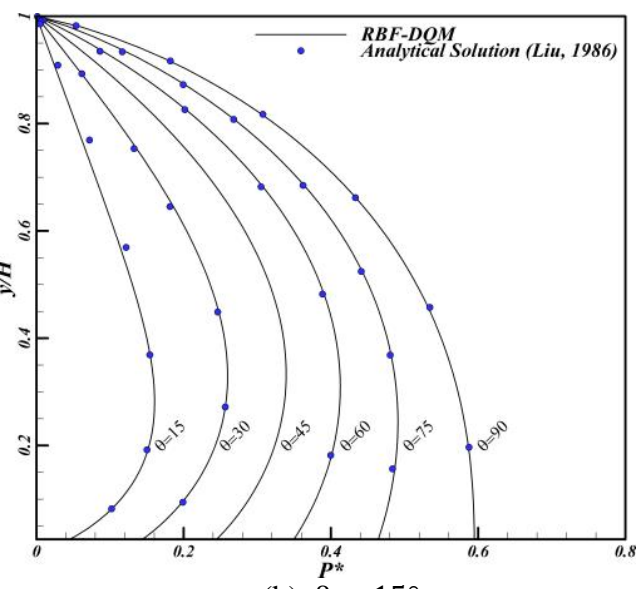

(b) $\beta=15^{\circ}$

Fig. 4. Distributions of Hydrodynamic Pressure on the Upstream Face of Dam in Case No.1.

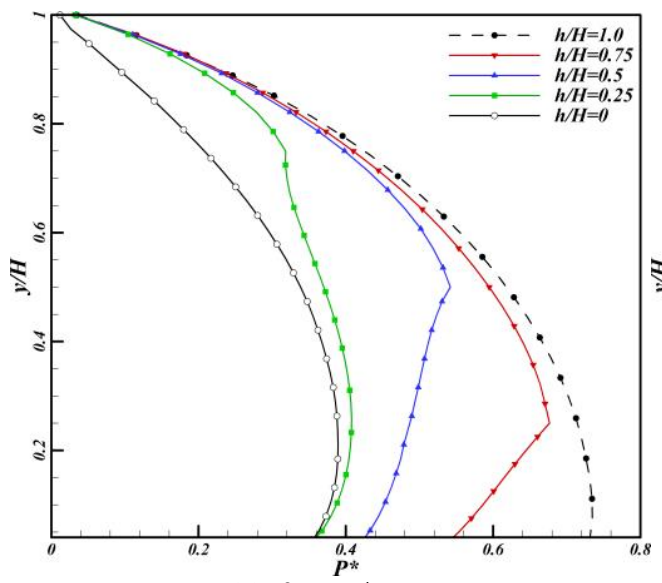

(a) $\theta=45^{\circ}$

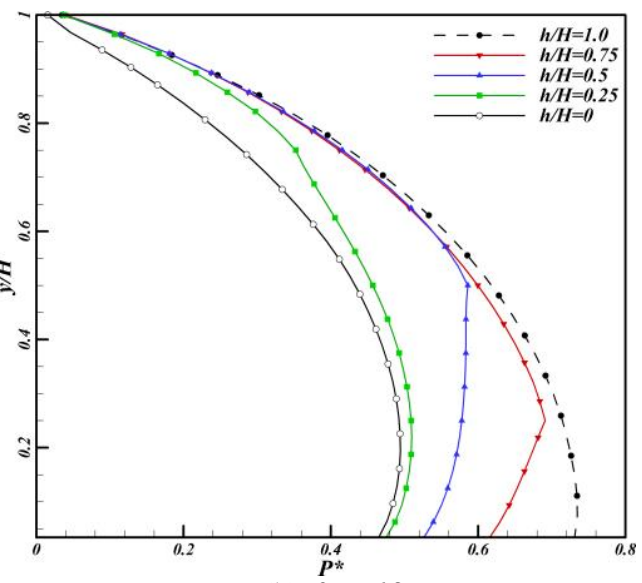

(b) $\theta=60^{\circ}$

Fig. 5. Distribution of Hydrodynamic Pressure on the Dam Face for Different Ratios of $h / H$ in Case No.1.

angles were calculated using RBF-DQM and the results were compared with the analytical solution. The steady-state distributions of dimensionless hydrodynamic pressure $P^{*}$ on the face of the dam for the reservoir bottom inclination, $\beta=5^{\circ}$ and $15^{\circ}$ and face angles, $\theta$ between $90^{\circ}$ (Vertical face) and $15^{\circ}$ from RBF-DQM results in comparison with the analytical solution are shown in Fig. 4.

The proximity between the results obtained from RBF-DQM and the analytical solution proves the excellent performance of the present model. The results also show significant increases in hydrodynamic pressure with increasing face inclination.

Also, with increasing the slope of the reservoir bottom, there is a decrease in hydrodynamic pressure on the dam face. This example with similar specifications and different values of $h / H$ ratio was also modeled to demonstrate the capability of the RBF-DQM in problems with more complex geometries. Numerical solutions are obtained for several dam geometries and illustrated in Fig. 5.

The typical pressure contour for $\theta=75^{\circ}$ and $\beta=$ $5^{\circ}$ form RBF-DQM results is shown in Fig. 6 . 


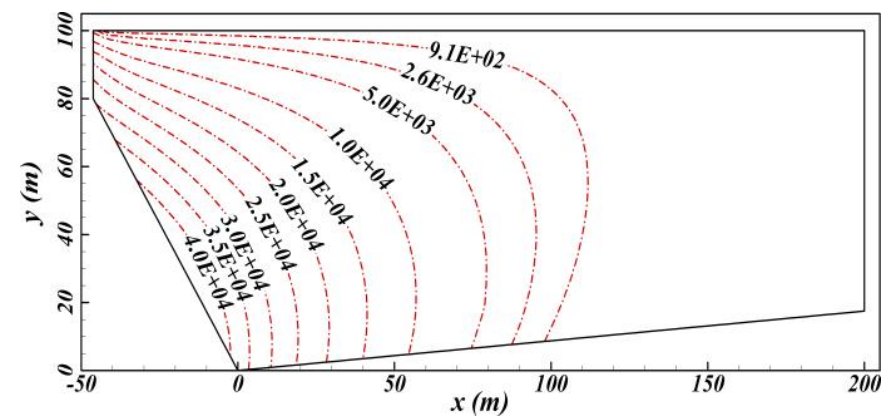

Fig. 6. Example of Pressure Contours in the Reservoir for Case No.1 when $\theta=75^{\circ}$ and $\beta=5^{\circ}$.

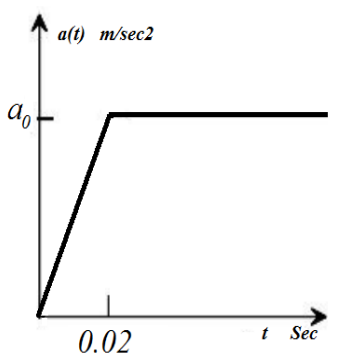

Fig. 7. Ramp Acceleration.

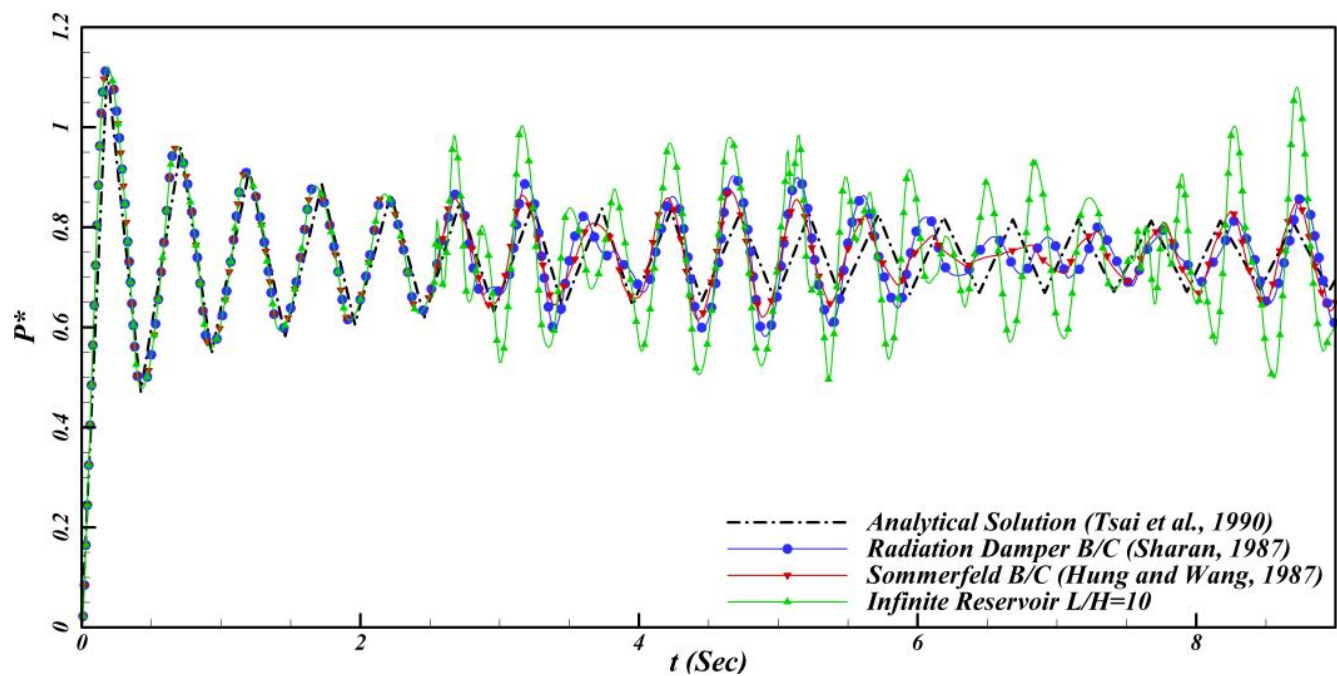

Fig. 8. Temporal Hydrodynamic Pressure in the Toe of the Dam for Case No.2.

\subsection{Case2: Vertical Face Rigid Dam}

To evaluate the capability of the present model in describing hydrodynamic behavior of the damreservoir system affected by dynamic acceleration load with more complicated boundary condition, a hypothetical vertical face rigid dam, with constant reservoir depth of $\mathrm{H}=180$, was considered. The reservoir truncated at $\mathrm{L}=10 \mathrm{H}$ away from the dam as the far-end boundary. The dam was affected by the ramp acceleration as shown in Fig. 7. The analysis was performed by neglecting the effect of the energy depreciation of the reservoir bottom and the effect of surface waves. Eqs. $(6,7$ and 8$)$ as various boundary conditions were implemented to model the far-end boundary of the infinite domain. With a similar approach to the previous case 388 mesh points were chosen as optimal mesh number to discretize the computational domain and the time increments were set to $\Delta \mathrm{t} 1.0 \mathrm{e}-4(\mathrm{~s})$ and RBF-DQM was applied to the problem. Tsai et al. (1990) has also provided an analytical solution for this problem under similar assumptions. The hydrodynamic pressure at the toe of the dam, under different boundary conditions is shown in Fig. 8 in comparison with the analytical solution. The hydrodynamic pressure on the dam face for different normalized depth is also obtained and the results for different expressions of boundary condition are compared with the analytical solution as shown in Fig. 9. The Comparison indicates good agreement between the obtained result and the analytical solution.

As figures show, the maximum hydrodynamic 
A. M. Behroozi and M. Vaghefi / JAFM, Vol. 13, No. 6, pp. 1759-1768, 2020.

Table 3 Variations of the Hydrodynamic Pressure on the Dam Face for Case No.2 Due to the Ramp Acceleration

\begin{tabular}{|c|c|c|c|c|}
\hline & $\begin{array}{c}\text { Analytical } \\
\text { y/H }\end{array}$ & $\begin{array}{c}\text { Radiation } \\
\text { Damper } \\
\text { Eq. (8) }\end{array}$ & Sommerfeld Eq. (7) & Infinite Reservoir Eq. (6) \\
\cline { 2 - 5 } & \multicolumn{5}{|c|}{$\mathrm{P}^{*}$ on the upstream face } \\
\hline 0 & $\mathbf{1 . 1 1 9 0 0}$ & 1.11300 & 1.10600 & 1.09300 \\
\hline 0.2 & 1.08786 & 1.08307 & 1.07500 & 1.06200 \\
\hline 0.4 & 0.97836 & 0.98114 & 0.97400 & 0.96500 \\
\hline 0.6 & 0.76875 & 0.78175 & 0.78500 & 0.48700 \\
\hline 0.8 & 0.48107 & 0.48807 & 0.48600 & 0.00000 \\
\hline 1.0 & 0.00000 & 0.00000 & 0.00000 & $2.0 \mathrm{e}-02$ \\
\hline $\mathrm{L}_{2}$ & - & $9.6 \mathrm{e}-03$ & $1.2 \mathrm{e}-02$ & \\
\hline
\end{tabular}

pressure occurred during the first half cycle and then oscillatory depreciated and the results have most consistency with the analytical solution while the Sharan boundary condition as formulated in (Sharan 1987) is applied to simulate the far-end of the reservoir. The hydrodynamic pressure induced on the different level of the dam face from RBF-DQM results in comparison with the analytical Solution for example No. 2 due to the Ramp Acceleration under different boundary conditions is summarized in Table 3.

The hydrodynamic behavior of this case was also determined using the present method when affected by simple harmonic acceleration, according to Eq. (22).

$\mathrm{a}(\mathrm{t})=\mathrm{a}_{0} \cos \cos (\mu \omega \mathrm{t})$

In which $\omega=\pi \mathrm{c} / 2 \mathrm{H}$ is the circular frequency of the reservoir and $\mu$ is the frequency ratio. Similar to the previous example, the effects of surface waves were neglected.

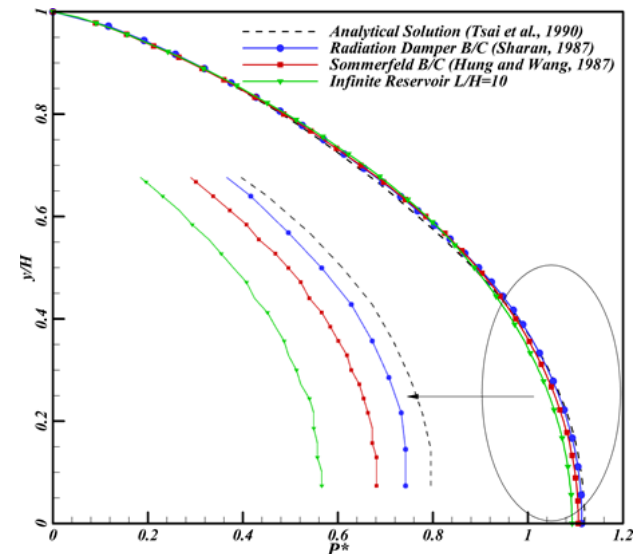

Fig. 9. Variation of Maximum Hydrodynamic Pressure on the Dam Face for Case No.2 Due to Ramp Acceleration.

The effect of energy dissipation on the reservoir bottom was studied by considering different values for $\alpha$ and implementing Eq. (4) as the reservoir bottom boundary condition at $(y=0)$. The meshing and size of the time increments were similar to the previous case and kept unchanged. The close-form analytical solution for the hydrodynamic pressures on dams under the similar harmonic load, proposed by Chopra (1967) is used to validate the results.

Fig. 10 illustrates the variations of steady-state hydrodynamic pressure on the dam face for $(\mu=1)$ and different values of $\alpha$ obtained from RBF-DQM in comparison with the analytical solution. The results, while confirming the excellent accuracy of RBF-DQM, indicate that increasing rigidity of the reservoir bottom, result significant increase of the hydrodynamic pressure on the dam face.

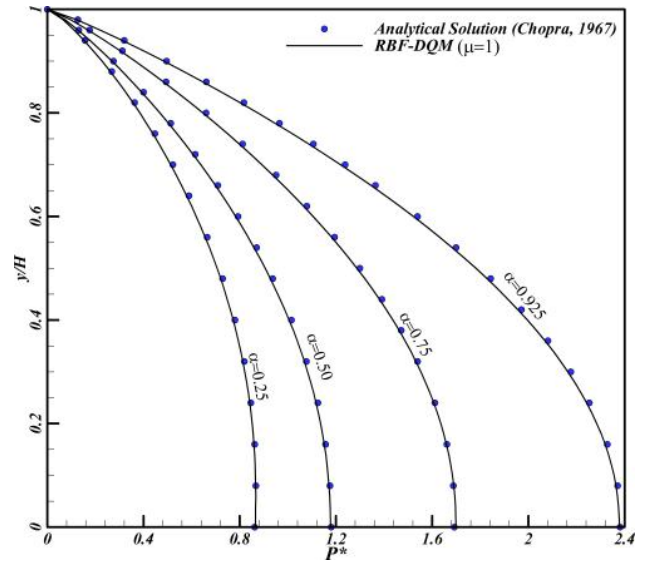

Fig. 10. Variation of Steady State Hydrodynamic Pressure on the Dam Face for Case No.2 Due to the Harmonic Acceleration.

Chopra (1967) also expressed the results in a spectrum of force $\mathrm{F}^{*}$ to investigate the effect of different $\alpha$ values on the hydrodynamic pressure on the dam face. In which $\mathrm{F}^{*}$ is defined as the proportion of the total hydrodynamic force, $F_{\text {dynamic }}$ (the steady-state hydrodynamic pressures integrated along the dam face) to the hydrostatic force, $F_{\text {static }}$ Chopra (1967). In a similar approach, the results for different values of $\mu$ and assuming a completely rigid reservoir bottom were obtained. The comparison of RBF-DQM results with graphs presented by Chopra (1967) is portrayed in Fig. 11. As the results show, the present model accurately obtains the hydrodynamic behavior of the system and maintains this ability in the range very close to the resonance state. 


\section{CONCLUSION}

In this work, a numerical model based on the Radial Basis Function Differential Quadrature method was introduced as a simple, efficient and accurate tool to determine hydrodynamic behavior of damreservoir-foundation system affected by earthquake acceleration loads. The mesh-free nature of this method was used for problems with complex geometries such as non-vertical face dam and sloping reservoir. The spatial derivatives were discretized using the present model and the temporal derivative is implicitly approximated using the finitedifference. Two practical problems affected by constant and time-dependent acceleration load were designed and simulated using the RBF-DQM. Several boundary conditions were implemented to model the truncated boundary in the far end of the reservoir and the effect of energy dissipation on the reservoir bottom.

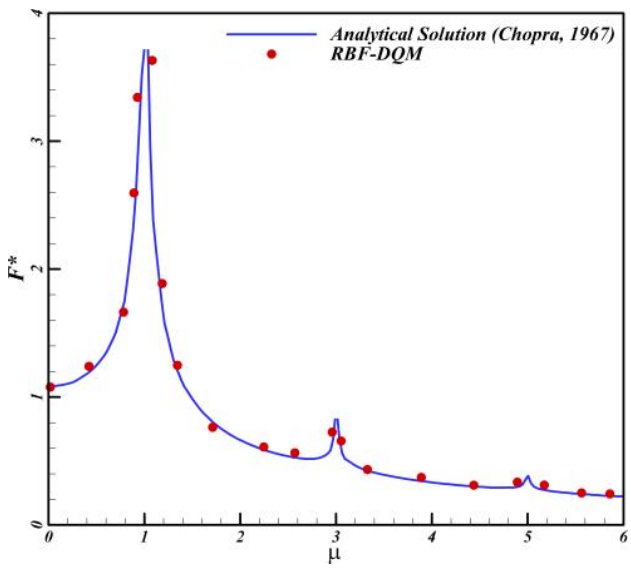

Fig. 11. Frequency Response Curves for Hydrodynamic Pressure on the Dam Face for Case No.2 Due to the Harmonic Acceleration.

Comparison of Obtained results with well-known analytical solution shows the accuracy of the RBFDQM.As can obviously see from results the reservoir geometry significantly influences the distribution of hydrodynamic pressures on a dam face and. The results also show that the Sharan type radiation damper boundary condition can model the far-end boundary more accurately than another boundary condition presented in literature.

\section{REFERENCES}

Abdollahi, M. and R. Attarnejad (2012). Dynamic analysis of dam-reservoir-foundation interaction using finite difference technique. Journal of Central South University 19(5), 1399-1410.

Bayraktar, A., E. Hançer and A. A. Dumanoğlu (2005). Comparison of stochastic and deterministic dynamic responses of gravity dam-reservoir systems using fluid finite elements. Finite Elements in Analysis and Design 41(14), 1365-1376.

Bellman, R. and J. Casti (1971). Differential quadrature and long-term integration. Journal of Mathematical Analysis and Applications 34(2), 235-238.

Belytschko, T. and Y. Y. Lu (1994). A variationally coupled FE-BE method for transient problems. International Journal for Numerical Methods in Engineering 37(1), 91-105.

Bouaanani, N. and F. Y. Lu (2009). Assessment of potential-based fluid finite elements for seismic analysis of dam-reservoir systems. Computers \& Structures 87(3-4), 206-224.

Bouaanani, N., P. Paultre and J. Proulx (2003). A closed-form formulation for earthquakeinduced hydrodynamic pressure on gravity dams. Journal of Sound and Vibration 261(3), 573-582.

Chen, B. F. and T. K. Hung (1993). Dynamic Pressure of Water and Sediment on Rigid Dam. Journal of Engineering Mechanics 119(7), 1411-1433.

Chopra, A. K. (1967). Hydrodynamic pressures on dams during earthquakes. Journal of the Engineering Mechanics Division 93 (6), 205224.

Chwang, A. T. and G. W. Housner (1978). Hydrodynamic pressures on sloping dams during earthquakes. Part 1. Momentum method. Journal of Fluid Mechanics 87(2), 335-341.

Czygan, O. and O. von Estorff (2002). Fluidstructure interaction by coupling BEM and nonlinear FEM. Engineering Analysis with Boundary Elements 26(9), 773-779.

Demirel, E. (2015). Numerical simulation of earthquake excited dam-reservoirs with irregular geometries using an immersed boundary method. Soil Dynamics and Earthquake Engineering 73, 80-90.

Ding, H., C. Shu and D. B. Tang (2005). Error estimates of local multiquadric-based differential quadrature (LMQDQ) method through numerical experiments. International Journal for Numerical Methods in Engineering 63(11), 1513-1529.

Estorff, O. Von and H. Antes (1991). On FEM-BEM coupling for fluid-structure interaction analyses in the time domain. International Journal for Numerical Methods in Engineering 31(6), 1151-1168.

Fenves, G. and A. K. Chopra (1984). Earthquake analysis of concrete gravity dams including reservoir bottom absorption and dam-waterfoundation rock interaction. Earthquake Engineering \& Structural Dynamics 12(5), 663-680.

Gogoi, I. and D. Maity (2006). A non-reflecting boundary condition for the finite element modeling of infinite reservoir with layered sediment. Advances in Water Resources 29(10), 1515-1527. 
Hall, J. F. and A. K. Chopra (1982). Twodimensional dynamic analysis of concrete gravity and embankment dams including hydrodynamic effects. Earthquake Engineering \& Structural Dynamics 10 (2), 305-332.

Hardy, R. L. (1971). Multiquadric equations of topography and other irregular surfaces. Journal of Geophysical Research 76(8), 19051915.

Howard, P. (2005). Partial differential equations in Matlab 7.0. University of Maryland, College Park (MD).

Humar, J. L. and A. M. Jablonski (1988). Boundary element reservoir model for seismic analysis of gravity dams. Earthquake Engineering \& Structural Dynamics 16(8), 1129-1156.

Hung, T. K. and M. H. Wang (1987). Nonlinear Hydrodynamic Pressure on Rigid Dam Motion. Journal of Engineering Mechanics 113(4), 482-499.

Küçükarslan, S., Coşkun, S. B. and B. Taşkin (2005). Transient analysis of dam-reservoir interaction including the reservoir bottom effects. Journal of Fluids and Structures 20(8), 1073-1084.

Liu, P. L. F. (1986). Hydrodynamic pressures on rigid dams during earthquakes. Journal of Fluid Mechanics 165(1), 131.

Lotfi, V., J. M. Roesset and J. L. Tassoulas (1987). A technique for the analysis of the response of dams to earthquakes. Earthquake Engineering \& Structural Dynamics 15(4), 463-489.

Maity, D. and S. K. Bhattacharyya (1999). Timedomain analysis of infinite reservoir by finite element method using a novel far-boundary condition. Finite Elements in Analysis and Design 32 (2), 85-96.

Medina, F. and J. Domínguez (1989). Boundary elements for the analysis of the seismic response of dams including dam-waterfoundation interaction effects. I. Engineering Analysis with Boundary Elements 6(3), 152157.

Nath, B. (1971). Coupled hydrodynamic response of a gravity dam. Proceedings of the Institution of Civil Engineers 48(2), 245-257.

Pelecanos, L., S. Kontoe and L. Zdravković (2013). Numerical modelling of hydrodynamic pressures on dams. Computers and Geotechnics $53,68-82$.

Saini, S. S., P. Bettess and O. C. Zienkiewicz (1978). Coupled hydrodynamic response of concrete gravity dams using finite and infinite elements. Earthquake Engineering \& Structural Dynamics 6(4), 363-374.

Sharan, S. K. (1987). Time-domain analysis of infinite fluid vibration. International Journal for Numerical Methods in Engineering 24(5), 945-958.
Shu, C. and Y. T. Chew (1997). Fourier expansionbased differential quadrature and its application to Helmholtz eigenvalue problems. Communications in Numerical Methods in Engineering 13(8), 643-653.

Shu, C. (2000). Differential Quadrature and Its Application in Engineering. London: Springer London.

Shu, C. and B. E. Richards (1992). Application of generalized differential quadrature to solve two-dimensional incompressible Navier-Stokes equations. International Journal for Numerical Methods in Fluids 15(7), 791-798.

Shu, C., H. Ding and K. S. Yeo (2004). Solution of partial differential equations by a global radial basis function-based differential quadrature method. Engineering Analysis with Boundary Elements 28 (10 SPEC. ISS.), 1217-1226.

Tsai, C. S., G. C. Lee and R. L. Ketter (1990). A semi-analytical method for time-domain analyses of dam-reservoir interactions. International Journal for Numerical Methods in Engineering 29(5), 913-933.

Wang, M. H. and T. K. Hung (1990). ThreeDimensional Analysis of Pressures on Dams. Journal of Engineering Mechanics 116(6), 1290-1304.

Wang, Y., Z. Hu and W. Guo (2019). Hydrodynamic pressures on arch dam faces with irregular reservoir geometry. Journal of Vibration and Control 25(3), 627-638.

Wang, Y., G. Lin and Z. Hu (2015). Novel nonreflecting boundary condition for an infinite reservoir based on the scaled boundary finiteelement method. Journal of Engineering Mechanics 141(5), 04014150.

Westergaard, H. M. (1933). Water pressures on dams during earthquakes. Trans. ASCE 95, 418-433.

Wilson, E. L. and M. Khalvati (1983). Finite elements for the dynamic analysis of fluid-solid systems. International Journal for Numerical Methods in Engineering 19(11), 1657-1668.

Wu, Y. L. and C. Shu (2002). Development of RBFDQ method for derivative approximation and its application to simulate natural convection in concentric annuli. Computational Mechanics 29(6), 477-485.

Zhao, M., X. Wang, P. Wang, X. Du and J. Liu (2019). Seismic water-structure interaction analysis using a modified SBFEM and FEM coupling in a frequency domain. Ocean Engineering 189, 106374.

Zhao, M., G. Zhang, P. Wang, X. Du and X. Zhang (2020). An accurate frequency-domain model for seismic responses of breakwater-seawaterseabed-bedrock system. Ocean Engineering 197, 106843. 